\title{
Pure T-cell-mediated rejection following kidney transplant according to the response to treatment in the era of antibody- mediated rejection
}

Hyunwook Kwon, Sung Shin, Duck Jong Han, Young Hoon Kim, Joo Hee Jung, Dong Hyun Kim, Youngmin Ko, Seong Jun Lim

Division of Transplantation, Department of Surgery, Asan Medical Center, University of Ulsan College of Medicine, Seoul, Korea

Background: Currently, we live in an era of antibody-mediated rejection (ABMR), while the effect of T-cell-mediated rejection (TCMR) on graft survival (GS) is gradually decreasing. However, acute pure TCMR is still described in histologic reports even a long time after transplant. The purpose of this study was to evaluate the effect of pure TCMR on GS according to the treatment response. We also performed molecular diagnosis using a molecular microscope diagnostic system (MMDx) to determine the accuracy of the histologic diagnosis of pure TCMR.

Methods: A total of 63 patients were divided into the non-responder group ( $n=22)$ and responder group ( $n=44)$ according to the response to rejection treatment. Molecular diagnosis and histologic diagnosis were conducted simultaneously on additional 23 patients.

Results: The rejection time since treatment was shorter in the non-responder group than in the responder group. In addition, the cumulative incidence of recurrent rejection after treatment was significantly higher in the non-responder group. The glomerular filtration rate (GFR) at biopsy, $\triangle$ GFR (baseline GFR-GFR at biopsy), TCMR within 1 year, t score, and interstitial fibrosis/tubular atrophy score were significant factors associated with the non-responder group. In comparison with the non-responder group, the responder group showed a significantly superior overall GS rate. Molecular assessment using MMDx showed a good correlation with the histologic diagnosis of ABMR but not TCMR.

Conclusions: Our study found that acute pure TCMR was a significant risk factor for graft failure in patients who did not respond to rejection treatment. Molecular analysis using MMDx showed that acute pure TCMR at 1 year after transplant in the histologic report must be interpreted cautiously.

Corresponding author: Young Hoon Kim

E-mail: gskyh@amc.seoul.kr

(c) The Korean Society for Transplantation

This is an Open Access article distributed under the terms of the Creative Commons Attribution Non-Commercial License (http://creativecommons.org/licenses/by-nc/4.0/) which permits unrestricted non-commercial use, distribution, and reproduction in any medium, provided the original work is properly cited. 